Doufexi, A., Prado-Miguelez, A., Armour, SMD., Nix, AR., \& Beach, MA. (2003). Use of space time block codes and spatial multiplexing using TDLS channel estimation to enhance the throughput of OFDM based WLANs. IEEE 57th Vehicular Technology Conference, 2003 (VTC 2003-Spring), 1, 704 - 708.

http://ieeexplore.ieee.org/search/srchabstract.jsp?arnumber=1207634 \&isnumber=27174\&punumber=8574\&k2dockey=1207634@ieeecnfs\& query $=\% 28+\% 28+$ doufexi $\% 3$ Cin $\% 3$ Emetadata+\%29+\%3Cand $\% 3 \mathrm{E}+$ $\% 28+$ armour\%3Cin\%3Emetadata+\%29+\%29\%3Cand\%3E+\%28+nix $\% 3$ Cin $\% 3$ Emetadata+\%29\&pos $=6$

Peer reviewed version

Link to publication record in Explore Bristol Research

PDF-document

University of Bristol - Explore Bristol Research

General rights

This document is made available in accordance with publisher policies. Please cite only the published version using the reference above. Full terms of use are available:

http://www.bristol.ac.uk/red/research-policy/pure/user-guides/ebr-terms/ 


\title{
Use of Space Time Block Codes and Spatial Multiplexing using TDLS Channel Estimation to Enhance the Throughput of OFDM based WLANs
}

\author{
Angela Doufexi, Arantxa Prado Miguelez, Simon Armour, Andrew Nix and Mark Beach \\ Centre for Communications Research, University of Bristol, UK
}

\{A.Doufexi, A.PradoMiguelez, Simon.Armour, Andy.Nix, M.A.Beach\}@bristol.ac.uk

\begin{abstract}
HIPERLAN/2, 802.11a, 802.11g and HiSWANa Wireless Local Area Networks (WLANs) employ Coded Orthogonal Frequency Division Multiplexing (COFDM) and provide data rates of up to $54 \mathrm{Mbps}$ in a $20 \mathrm{MHz}$ bandwidth. In this paper, space-time block coding (STBC) and spatial multiplexing MIMO techniques are considered as a means of enhancing the performance of COFDM WLANs. To perform channel estimation for multiple transmit and receive antennas, it is necessary to make transmitted pilot signals orthogonal in space and time. In this paper, this was achieved using a Hadamard design. A time domain least square channel estimator was used to achieve high performance. Packet Error Rate (PER) performance results for the case of $2 \mathrm{Tx}$ and $2 \mathrm{Rx}$ antennas are presented and throughput enhancements are evaluated. It is observed that STBC doubles the link throughput for low SNR values. As expected, multiplexing achieves significant throughput enhancements at high SNR values at the expense of additional complexity.
\end{abstract}

\section{INTRODUCTION}

At present, Wireless Local Area Networks (WLANs) supporting broadband multimedia communication are being standardized and rolled out around the world. Standards include HIPERLAN/2 [1], defined by ETSI BRAN, 802.11a [2] and 802.11g [3] defined by the IEEE and HiSWANa [4] defined by ARIB MMAC. These systems all provide channel adaptive data rates up to $54 \mathrm{Mbps}$ (in a $20 \mathrm{MHz}$ channel spacing) in the $5 \mathrm{GHz}$ and $2.4 \mathrm{GHz}$ (for the case of $802.11 \mathrm{~g}$ ) radio bands.

In order to enhance performance, multiple transmit and receive antennas can be used to provide diversity or increase capacity. Alamouti [5] proposed a simple 2 transmit 2 receive antenna Space Time Block Coding (STBC) algortihm. It requires modest additional complexity and is suitable for the slow fading environments in which WLANs are deployed. STBC can enhance performance by exploiting spatial diversity. This is particularly useful in the case where the delay spread of the environment is low (i.e. low frequency diversity). For these reasons, STBC techniques have been examined to enhance the PER performance of WLANs [6-8]. Spatial multiplexing [17] relies on transmitting independent data streams from each transmit antenna. These data streams can be multiplexed from the incoming source stream. If $N$ transmit and receive antennas are present then data can be sent at $N$-times the rate of a standard terminal. Spatial multiplexing exploits the benefits of the MIMO channel to enhance the rate at which data is sent, rather than enhancing the reliability of its detection. Channel coding is commonly applied prior to the spatial multiplexing process. This coding is normally applied at bit level prior to modulation and multiplexing. In this paper, link throughput results will be presented for the case of 2 transmit and 2 receive antennas with time domain Least Squares (LS) channel estimation [9] performed for both STBC and spatial multiplexing. Results show that link throughput is significantly enhanced in both cases.

\section{WLAN PHYSICAL LAYERS}

The physical layers of HIPERLAN/2, 802.11a, 802.11g and HiSWANa are based on the use of coded Orthogonal Frequency Division Multiplexing (COFDM). COFDM is used to combat frequency selective fading and to randomize the burst errors caused by a wideband-fading channel. The OFDM modulation is implemented by means of an inverse FFT. 48 data symbols and 4 pilots are transmitted in parallel in the form of one OFDM symbol. In order to prevent ISI, a guard interval is implemented by means of a cyclic extension. When the guard interval is longer than the excess delay of the radio channel, ISI is eliminated. In that case, the signal received after the FFT can be written as:

$$
y_{k}=H_{k} x_{k}+n_{k}
$$

where $y_{k}$ is the received signal at a given subcarrier $k, H_{k}$ is the frequency response of the channel at the $k^{\text {th }}$ subcarrier, $n_{k}$ represents complex Additjve White Gaussian Noise (AWGN), and $x_{k}$ is the transmitted signal at subcarrier $k$.

Table I: WLANs transmission modes

\begin{tabular}{|c|c|c|c|}
\hline Mode & Modulation & $\begin{array}{c}\text { Coding } \\
\text { Rate R }\end{array}$ & $\begin{array}{c}\text { Bit rate } \\
{[\text { Mbit/s] }}\end{array}$ \\
\hline 1 & BPSK & $1 / 2$ & 6 \\
\hline 2 & BPSK & $3 / 4$ & 9 \\
\hline 3 & QPSK & $1 / 2$ & 12 \\
\hline 4 & QPSK & $3 / 4$ & 18 \\
\hline $5(802.11 \mathrm{a} / \mathrm{g})$ & 16QAM & $1 / 2$ & 24 \\
\hline $5(\mathrm{H} 2, \mathrm{Ha})$ & $16 \mathrm{QAM}$ & $9 / 16$ & 27 \\
\hline 6 & $16 \mathrm{QAM}$ & $3 / 4$ & 36 \\
\hline 7 & $64 \mathrm{QAM}$ & $3 / 4$ & 54 \\
\hline $8(802.11 \mathrm{a} / \mathrm{g})$ & $64 \mathrm{QAM}$ & $2 / 3$ & 48 \\
\hline
\end{tabular}

Importantly, the physical layer provides several modes (Table I) each with a different coding and modulation configuration. These are selected by a link adaptation scheme. A simple approximation of the link throughput when retransmission is employed is given by: Throughput $=R(1$ $P E R$, where $R$ and $P E R$ are the bit rate and packet error rate for a specific mode respectively. In the case where a simple link adaptation scheme is used, the mode with the highest 
throughput can be chosen for each instantaneous SNR value. Physical layer details can be found in $[14,15]$.

\section{SPACE TIME Block CODES}

In [5] Alamouti proposed a simple transmit diversity scheme which was generalized by Tarokh [10] to form the class of Space-Time Block Codes (STBC). These codes achieve the same diversity advantage as maximal ratio receive combining (allowing for a $3 \mathrm{~dB}$ degradation for the case of 2 $\mathrm{Tx}$ antennas due to power normalization) [11]. In [12], Lee and Williams applied a $2 \mathrm{Tx}-1 \mathrm{Rx}$ antenna, transmit diversity scheme to OFDM in order to achieve diversity gain over frequency selective fading channels.

In Alamouti's encoding scheme two signals are transmitted simultaneously from the 2 transmit antennas. The transmission matrix is given by $[5,10-13]$ :

$$
\mathrm{X}=\left[\begin{array}{cc}
x_{1, k} & -x_{2, k}^{*} \\
x_{2, k} & x_{1, k}^{*}
\end{array}\right]
$$

where in the case of OFDM, $x_{l, k}, x_{2, k}$ are the transmitted signals at a given subcarrier $k$ (from two consecutive OFDM symbols) before being input to the IFFT and after the serial to parallel conversion (S/P) of the QAM modulated data [12]. At the first antenna, for the $k^{\text {th }}$ subcarrier, $x_{l, k}$ is transmitted during the first symbol period followed by $-x_{2, k}{ }^{*}$ in the second symbol period. At the second antenna, $x_{2, k}$ is transmitted during the first symbol period followed by $x_{1, k}{ }^{*}$ in the second symbol period.

At receive antenna 1 , the cyclic prefix removal and FFT, the received signals are given by $[5,12]$ :

$$
\begin{gathered}
y_{l, k}=x_{l, k} H_{l, k}+x_{2, k} H_{2, k}+n_{1, k} \\
y_{2, k}=-x_{2, k} H_{l, k}+x_{l, k}{ }^{*} H_{2, k}+n_{2, k}
\end{gathered}
$$

where $n_{l, k} n_{2, k}$ represent AWGN and $H_{l, k}$ and $H_{2, k}$ are the frequency responses, at a given subcarrier $k$, of the channels between $\mathrm{TxI}$ and $\mathrm{RxI}$ and $\mathrm{T} \times 2$ and $\mathrm{Rx} 1$ respectively. It is assumed that the channel responses are uncorrelated and constant during the period of two OFDM symbols. The latter is reasonable for the OFDM parameters specified for the WLANs. After channel estimation, the channel parameters are known to the receiver, and the signals $y_{1, k}, y_{2, k}$ can be combined at the receiver according to [12]:

$$
\begin{gathered}
s_{l, k}=y_{l, k} H_{l, k}^{*}+y_{2, k}^{*} H_{2, k} \\
s_{2, k}=y_{l, k} H_{2, k}^{*}-y_{2, k}^{*} H_{l, k}
\end{gathered}
$$

Substituting for $y_{1, k}, y_{2, k}$ the combined signals can be written as $[5,12]$ :

$$
\begin{aligned}
& s_{l, k}=x_{I, k}\left(\left|H_{l, k}\right|^{2}+\left|H_{2, k}\right|^{2}\right)+n_{l, k} H_{l, k}{ }^{*}+n_{2, k}{ }^{*} H_{2, k} \\
& s_{2, k}=x_{2, k}\left(\left|H_{I, k}\right|^{2}+\left|H_{2, k}\right|^{2}\right)+n_{l, k} H_{2, k}{ }^{*}-n_{2, k}{ }^{*} H_{l, k}
\end{aligned}
$$

In order to perform soft decision Viterbi decoding, the Channel State Information of both channels and for all subcarriers $\left(H_{l, k}, H_{2, k}\right)$ is passed to the decoder in order to calculate the metric.
For the case of $1 \mathrm{Rx}$ antenna, the above scheme is similar to that of two branch maximal ratio receive combining (MRRC). For the case of $2 \mathrm{Rx}$ antennas, the signal from the two receivers are combined and the scheme performs similar to four branch MRRC.

\section{SPATIAL Multiplexing}

Layered space time (LST) architectures proposed in [17] represent one of a number of different MIMO techniques for achieving capacity enhancement. In this paper the simplest LST architecture, known as vertical Bell Laboratories layered space time (V-BLAST), has been implemented in order to compare its performance against the STBC algorithm.

At the transmitter, the binary data, after being encoded by the convolutional encoder and interleaved by the specified algorithms in the HIPERLAN/2 standard, are mapped to the constellation set and then simply de-multiplexed into 2 symbol streams in order to be transmitted by each of the 2 antennas.

At the receiver, the cyclic prefix is removed from the received streams and then transformed by the FFT back to the frequency domain as in the STBC case. The detector algorithm consists of a maximum likelihood (ML) detector where the probability of the possible transmitted symbols is calculated as follows:

$$
p(x \mid r)=\sum^{\Lambda} \exp \left(-\frac{\|r-H x\|^{2}}{2 \sigma^{2}}\right)
$$

where $x$ is the vector of all possible transmitted symbols, ie. $\left[\mathrm{x}_{1} \ldots \mathrm{x}_{\mathrm{j}}\right]^{\mathrm{T}}$, the subscript $j$ represents the transmitting antenna and $\Lambda$ is the antenna array constellation set. This calculation is computed for every $k t h$ subcarrier in the OFDM symbol. A soft decision Viterbi decoder is used where loglikelihood ratios $(L L R)$ are fed into the decoder. The reason for this is that $L L R$ can be easily calculated from the output symbol probabilities of the detector as shown by Equation 7 and 8 . The bit probabilities can be calculated from the symbol probabilities as:

$$
p\left(b_{n}\right)=\sum_{j=1}^{M} p\left(x_{j} \mid b_{n}\right)
$$

where $n$ is the bit position corresponding to the $j$ th symbol in a constellation of size $M$. The $L L R$ can be calculated as:

$$
L L R\left(b_{n}\right)=\ln \frac{p\left(b_{n}=+1\right)}{p\left(b_{n}=-1\right)}
$$

where the probability of a bit $b$ can be computed from the symbol probabilities.

\section{Channel Estimation}

To perform channel estimation, OFDM based WLANs use two consecutive pilot OFDM symbols in the preamble. In order to avoid adding additional overhead when 2 transmit and 2 receive antennas are employed, two pilot OFDM symbols will be transmitted from each transmit antenna simultaneously. In order for this to work, the pilot symbols must be orthogonal. One family of pilots whose orthogonality 
is obtained jointly in space and time dimensions is the set of Orthogonal Space-Time Pilot Matrices (OSTPM) [9]. STBC matrices and Hadamard matrices contain the desired properties [9]. In this paper, a Hadamard matrix is used to transmit the real pilot symbols according to;

$$
\mathrm{X}=\left[\begin{array}{cc}
x_{1, k} & x_{1, k} \\
x_{1, k} & -x_{1, k}
\end{array}\right]
$$

Using this set of pilots the channels can be easily separated at the receiver by a linear combination of the received pilots [9]. Hence, the channel parameters can be computed independently for each transmit antenna according to:

$$
\begin{gathered}
r_{l, k}=x_{l, k} H_{l, k}+x_{l, k} H_{2, k}+n_{l, k} \\
r_{2, k}=x_{l, k} H_{l, k}-x_{l, k} H_{2, k}+n_{2, k} \\
s_{l, k}=r_{l, k}+r_{2, k}=2 x_{l, k} H_{l, k}+n_{l, k}+n_{2, k} \\
s_{2, k}=r_{l, k}-r_{2, k}=2 x_{l, k} H_{2, k}+n_{l, k}-n_{2, k}
\end{gathered}
$$

where $r_{l, k}, r_{2, k}$ are the received pilots. This way, the channel parameters can be directly estimated in the frequency domain in the least square (FD LS) sense.

However, OFDM based WLAN standards use 12 virtual subcarriers at the edge of the band and only $K=52$ subcarriers are used for transmission. Additionally, in order to prevent ISI, a guard interval, $G$, of 16 samples is used. This means that the channel impulse response is expected to have less than 16 coefficients. If we denote $F^{|K . G|}$ to represent the truncated 64 point Fourier matrix, in which the rows correspond to the $K$ observed subcarriers and only the first $G$ columns are kept, equation (11) can be written as:

$$
\begin{aligned}
& S_{I}=2 X_{1} H_{1}+N_{1}+N_{2}=2 X_{I} F^{[K, G I} h_{l}^{[G]}+N_{I}+N_{2} \\
& S_{2}=2 X_{1} H_{2}+N_{I}+N_{2}=2 X_{I} F^{[K, G]} h_{2}^{i G]}+N_{I}+N_{2}
\end{aligned}
$$

where $X_{1}=\operatorname{diag}\left(\left\{x_{1, k}\right\}_{k=0: K-l}\right), N_{l}, N_{2}$ are AWGN vectors, $H_{l}=\left[H_{1,0} \ldots H_{1, K-I}\right]^{\mathrm{T}}$ and $H_{2}=\left[H_{2,0} \ldots H_{2, K-I}\right]^{\mathrm{T}}$ and $h_{l}{ }^{|G|}, h_{2}{ }^{\mid O l}$ are the time domain channel impulse response vectors.

The time domain least square (TD LS) estimator of the frequency coefficients is given by:

$$
\begin{aligned}
& H_{l, T D} L S=F^{[K, G]}\left[2 X_{1} F^{[K, G\}}\right]^{\dagger} S_{I} \\
& H_{2, T D} L S=F^{[K, G]}\left[2 X_{1} F^{(K, G)}\right]^{\dagger} S_{2}
\end{aligned}
$$

where the Moore-Penrose pseudo-inverse of a matrix $\mathrm{A}$ is denoted $A^{\dagger}$. The TD LS channel estimator requires some additional complexity since it is obtained by projecting the FD LS solution in the time domain by using $\left(F^{[K, G]}\right)^{\dagger}[9]$. However, performance is significantly improved, as can be observed from the results presented in the next section. Equation (13), can be seen as a form of filtering of the channel coefficients, since we know that the channel impulse response has less than 16 coefficients.

\section{STBC PERFORMANCE RESULTS}

A detailed PHY layer software simulator of HIPERLAN/2 and $802.11 \mathrm{a} / \mathrm{g}$ has been developed previously by the authors [15]. This simulator was enhanced here by adding STBC and spatial multiplexing together with different channel estimation options.

Figure 1, shows the PER performance of mode 3 versus SNR with STBC for the cases of time domain least square (TD LS) estimation, frequency domain least square (FD LS) estimation and ideal channel estimation. Results are shown for 2 transmit and 1 receive antenna and for 2 transmit and 2 receive anternas for a packet size of 54 bytes (HIPERLAN/2) for channel model A as specified by ETSI BRAN [16]. It can be observed that the performance of the TD LS estimation is close to the ideal channel estimation case, with $\sim 2 \mathrm{~dB}$ degradation for the case of $2 \mathrm{Tx}$ and $2 \mathrm{Rx}$ at a PER $=10^{-3}$. The FD LS estimation results in $\sim 4 \mathrm{~dB}$ degradation in performance relative to the ideal channel estimation. Thus, TD LS channel estimation will be used for the remainder of the results presented in this paper. Note that FD LS has a lower computational complexity compared to TD LS since fewer domain transformations are required.

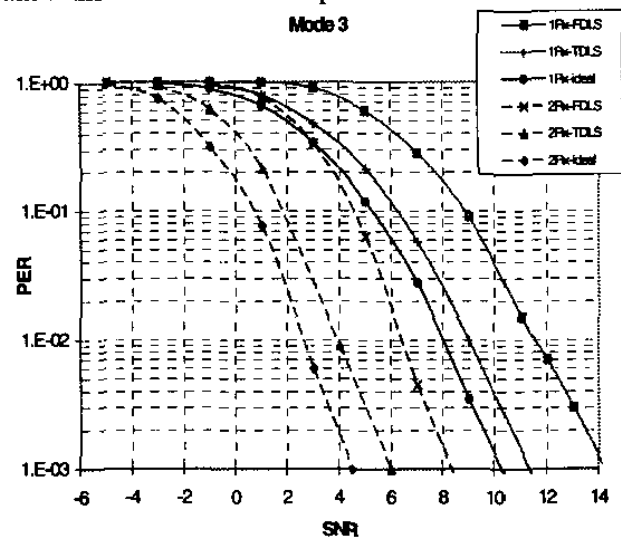

Figure 1: PER Performance for mode 3 using STBC with 2Tx, IRx and 2Tx, $2 \mathrm{Rx}$ antennas for FD LS and TD LS channel estimation.

Figures 2 and 3 show the PER performances for all modes for the cases of a standard HIPERLAN/2 modem (with 1Tx and $(R x)$ and for the case of $2 T x$ and $2 R x$ antennas with STBC respectively. It can be seen that performance is significantly enhanced, providing gains of 8.5-10.5 dB depending on the mode (and channel model). Modes 2, 4 and 6 (3/4 rate modes with puncturing) exhibit the most benefit. Table II, summarises the STBC gains achieved at a PER $=10^{-2}$. Note that all the link throughput enhancements shown for HIPERLAN $/ 2$ are valid for the identical modes of $802.11 \mathrm{a} / \mathrm{g}$ and HISWANa WLANs and that similar gains can be expected for the similar but non-identical modes of the other standards.

Figures 4 and 5 show the link throughput for the case of a standard HIPERLAN/2 modem and for the case of $2 \mathrm{~T} x$ and $2 R x$ antennas with STBC respectively. A simple link adaptation scheme where the mode with the highest throughput is chosen for each instantaneous SNR value is assumed. Table III, summarises the throughput achieved with and without space time block codes. It can be seen that STBC doubles the link throughput for SNR values up to $\sim 20 \mathrm{~dB}$ and 
throughput is higher than $50 \mathrm{Mbps}$ for SNR values higher that $16 \mathrm{~dB}$.

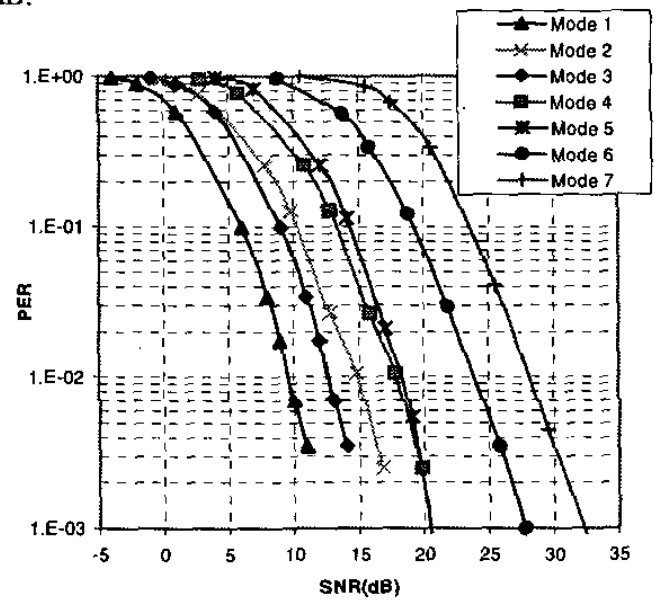

Figure 2: PER Performance of a standard HIPERLAN/2 system.

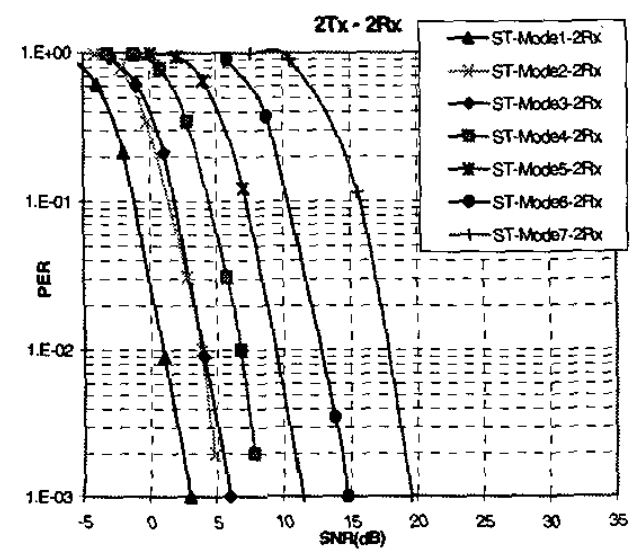

Figure 3: PER Performance using STBC with 2Tx, 2Rx antennas and TD LS channel estimation.

Table II: STBC Gain at PER $=10^{-2}$

\begin{tabular}{|c|c|}
\hline Mode & Gain (dB) \\
\hline 1 & 8.5 \\
\hline 2 & 10.5 \\
\hline 3 & 8.5 \\
\hline 4 & 10.5 \\
\hline 5 & 9 \\
\hline 6 & 9.5 \\
\hline 7 & 10 \\
\hline
\end{tabular}

Table III: Link Throughput with STBC

\begin{tabular}{|c|c|c|}
\hline SNR(dB) & 1Tx, 1Rx antenna & 2Tx, 2Rx antennas \\
\hline 0 & $2 \mathrm{Mbps}$ & 7 Mbps \\
\hline 5 & $7 \mathrm{Mbps}$ & $16 \mathrm{Mbps}$ \\
\hline 10 & $11 \mathrm{Mbps}$ & $26 \mathrm{Mbps}$ \\
\hline 15 & $22 \mathrm{Mbps}$ & $45 \mathrm{Mbps}$ \\
\hline 20 & $34 \mathrm{Mbps}$ & $54 \mathrm{Mbps}$ \\
\hline 25 & $51 \mathrm{Mbps}$ & $54 \mathrm{Mbps}$ \\
\hline
\end{tabular}

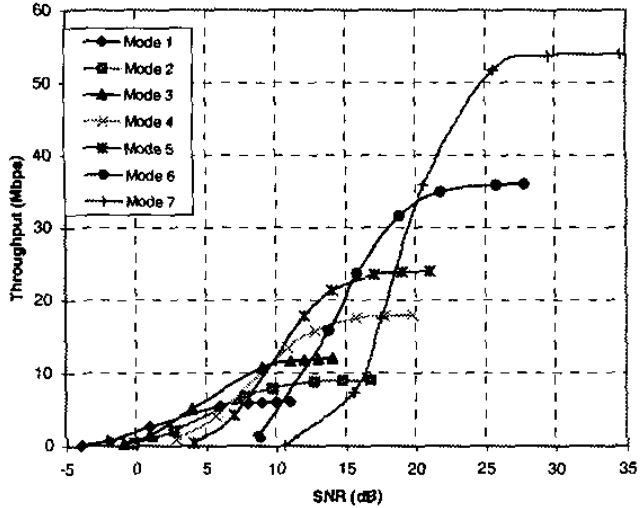

Figure 4: Link Throughput for HIPERLAN/2

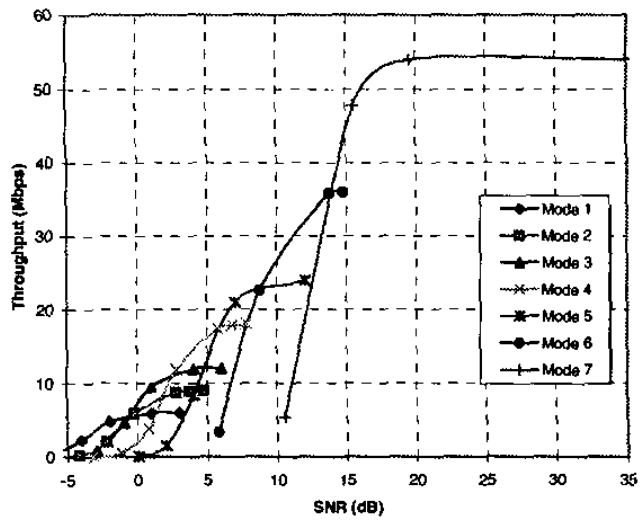

Figure 5: Link Throughput with STBC, 2Tx, 2Rx.

VII. SPATIAl MUlTipleXing PERformanCe ResUlts

Figure 6 shows the spatial multiplexing PER performance for the higher transmission modes using the different estimation techniques in a 2 transmit 2 receive antenna array.

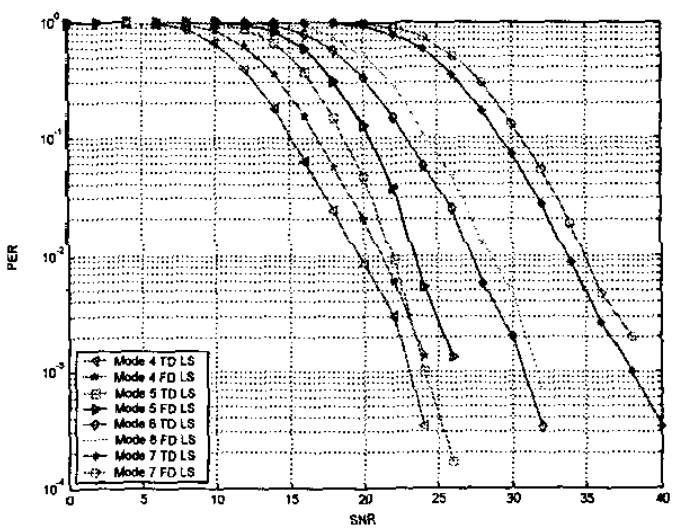

Figure 6: PER Performance using VBLAST with 2Tx, 2Rx antennas and TD LS and FD LS channel estimation.

The results show that the performance is enhanced for all modes when the TD LS channel estimator is used rather than 
the FD LS case, as seen previously with STBC. At a target PER of $10^{-3}$ using TD LS a gain of $1 \mathrm{~dB}$ is obtained over FD LS. This gain is constant over all transmission modes.

The link throughput was also calculated for the VBLAST architecture for all 7 transmission modes specified by the standard and the results are shown in Figure 7. Comparing Tables III and IV, it can be seen that the link throughput has been doubled at high signal to noise ratios compared to the STBC because VBLAST has been designed in order to enhance capacity rather than enhance SNR performance. However, STBC have a larger throughput at lower signal to noise ratios (SNR $\leq 20 \mathrm{~dB}$ ) because they have better PER performance due to the diversity gain.

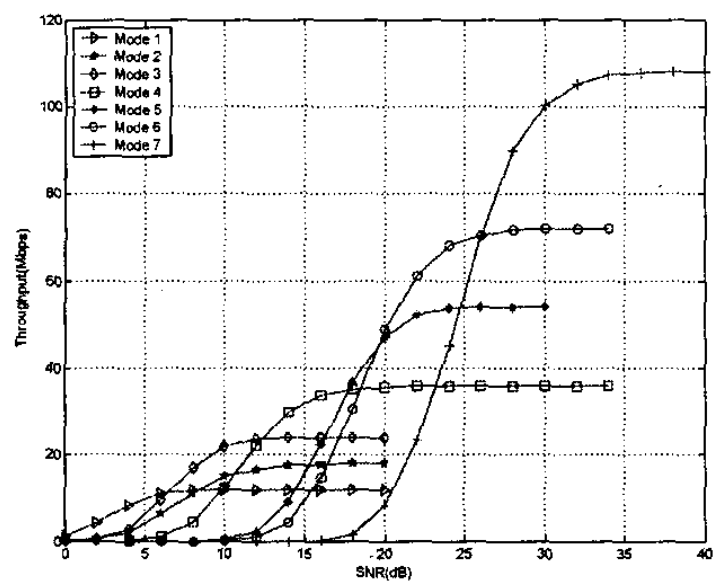

Figure 7: Link Throughput with VBLAST, 2Tx, 2Rx

Table IV: Link Throughput with VBLAST

\begin{tabular}{|c|c|c|}
\hline SNR(dB) & 1Tx, 1Rx antenna & 2Tx, 2Rx antennas \\
\hline 0 & $2 \mathrm{Mbps}$ & $2 \mathrm{Mbps}$ \\
\hline 5 & $7 \mathrm{Mbps}$ & $10 \mathrm{Mbps}$ \\
\hline 10 & $11 \mathrm{Mbps}$ & $22 \mathrm{Mbps}$ \\
\hline 15 & $22 \mathrm{Mbps}$ & $32 \mathrm{Mbps}$ \\
\hline 20 & $34 \mathrm{Mbps}$ & $49 \mathrm{Mbps}$ \\
\hline 25 & $51 \mathrm{Mbps}$ & $69 \mathrm{Mbps}$ \\
\hline 30 & $54 \mathrm{Mbps}$ & $100 \mathrm{Mbps}$ \\
\hline 35 & $54 \mathrm{Mbps}$ & $107 \mathrm{Mbps}$ \\
\hline
\end{tabular}

\section{VIII.CONCLUSIONS}

In this paper, space-time block coding and spatial multiplexing techniques were considered as a means of enhancing the performance and throughput of OFDM WLANs. To perform channel estimation for multiple transmit and receive antennas, a Hadamard design for the pilots was employed to achieve orthogonality in space and time. A time domain least square channel estimator was used to achieve superior performance. PER performance results for the case of $2 T x$ and $2 R x$ antennas and throughput enhancements were presented. When STBC was employed, gains between 8.5 . $10.5 \mathrm{~dB}$ were observed at a PER of $10^{-2}$. On the other hand, when spatial multiplexing was employed the capacity is enhanced. It was shown that at low SNRs (up to 20dB) STBC achieves greater capacity than spatial multiplexing because the orthogonal codes exploit the diversity of the system. However at higher SNR (SNR > 20dB), spatial multiplexing perform's better and can provide data rates in excess of $100 \mathrm{Mbps}$. It is important to point out that the ML detector has a greater complexity due to the number of symbol searches across the transmitting antennas while STBC has a much lower implementation complexity.

\section{ACKNOWLEDGEMENTS}

The authors wish to acknowledge the financial support of the IST ROMANTIK project (funded by the European Union) and A. Prado would also like to thank Toshiba Research Europe Limited (TREL).

\section{REFERENCES}

[1] ETSI, "Broadband Radio Access Networks (BRAN); HIPERLAN type 2 technical specification; Physical (PHY) layer," August 1999. <DTS/BRAN-0023003> V0.k

[2] IEEE Std 802.11a/D7.0-1999, Part11: Wireless L.AN Medium Access Control (MAC) and Physical Layer (PHY) specifications: High Speed Physical Layer in the $5 \mathrm{GHz}$ Band.

[3] IEEE Std 802.11 g/D1.1-2001, Part11: Wireless LAN Medium Access Control (MAC) and Physical Layer (PHY) specifications: Further Higher-Speed Physical Layer Extension in the $2.4 \mathrm{GHz}$ Band

[4] T.Aramaki, "HiperLAN2/HISWANa Hamonization, Difference Between HiperLAN2 and HISWANa," Presented to HiperLAN2 Global Forum JTF\#1, Sophia Antipolis (France), June 2001.

[5] M.Alamouti, "A simple transmit diversity technique for wireless communications", IEEE Journal on Selected Areas in Communications, Vol. 16, No.8, October 1998.

[6] M.Pauli, B.Wegmann, A.Krämling, A.Kadelka, T.Bing, "First Performance Results of BRAIN", IST Summit, Galway, Ireland, October 2000.

[7] A.Prado Migualez, A.Nix, J.P.McGeehan, "Receive Diversity versus Space Time Block Codes in IEEE 802.11a and ETSI HIPERLAN/2", VTC 2002 Fall, Vancouver.

[8] M. Gidlund, "Enhancement of Hiperlan/2 System using Space-Time Coding", European Wireless 2002, Florence, February 2002

[9] A.Ribeiro Dias, S Rouquette, K.Gosse, "MTMR Channel Estimation and Pilot Design in the Context of Space Time Block Coded OFDMBased WLANs", IST Summit, Thessaloniki, June 2002.

[10] V.Tarokh, H.Jafarkhani, A.R.Calderbank, "Space-time block coding for wireless communications: performance results", IEEE Joumal on Selected Areas in Communications, Vol. 17 No. 3, March '99, pp.451460.

[11] B. Vucetic, "Space-Time Codes for High Speed Wireless Communications", Course on Space Time Codes, King's College London, November 2001.

[12] K.F.Lee, D.B.Williams, "A space-time coded transmitter diversity technique for frequency selective fading channels", Sensor Aray and Multichannel Signal Processing Workshop, 2000, pp. 149 -152.

[13] A.F.Naguib, N.Seshadri, A.R.Calderbank, "Increasing data rate over wireless channels", IEEE Signal Processing Magazine Vol.17 No.3, May 2000 , pp.76-92.

[14] A.Doufexi, S.Armour, P.Karlsson, A.Nix, D.Bull, "Throughput Performance of WLANs Operating at $5 \mathrm{GHz}$ Based on Link Simulations with Real and Statistical Channels," IEEE VTC'01 Spring (Rhodes).

[15] A. Doufexi, S. Armour, P. Karlsson, M. Butler, A. Nix, D. Bull, J. McGeehan, "A Comparison of the HIPERLAN/2 and IEEE 802.11a Wireless LAN Standards," IEEE Communications Magazine, May 2002, Vol. 40, No. 5.

[16] J.Medbo, P. Schramm "Channel Models for HIPERLAN/2" ETS1BRAN document no. 3ERI085B, 1998.

[17] G.J.Foschini,'Layered space-time architectures for wireless communications in a fading environment when using multiple antennas",In Bell Labs Tech. 1996 